\title{
Improvement of bowel movements among people with a sedentary lifestyle after prebiotic snack supply - preliminary study
}

\author{
Ewa Stachowska1 ${ }^{1}$, Dominika Maciejewska ${ }^{1}$, Joanna Palma ${ }^{1}$, Dominika Jamioł-Milc ${ }^{1}$, Małgorzata Szczuko', \\ Wojciech Marlicz ${ }^{2}$, Ewa Wunsch ${ }^{3}$, Karolina Skonieczna-Żydecka ${ }^{1}$ \\ ${ }^{1}$ Department of Human Nutrition and Metabolomics, Pomeranian Medical University, Szczecin, Poland \\ 2Department of Gastroenterology, Pomeranian Medical University, Szczecin, Poland \\ ${ }^{3}$ Translational Medicine Group, Pomeranian Medical University, Szczecin, Poland
}

Gastroenterology Rev 2022; 17 (1): 73-80

DOI: https://doi.org/10.5114/pg.2021.108985

Key words: prebiotics, dietary fibre, microbiota, constipation, clinical trial.

Address for correspondence: Prof. Ewa Stachowska; Department of Human Nutrition and Metabolomics, Pomeranian Medical University, Szczecin, Poland, e-mail: ewast@pum.edu.pl

\begin{abstract}
Introduction: Dietary fiber is one of the most important components of food. Fulfilling regulatory and nutritional functions for the intestinal microbiota, it appears to be an essential ingredient for people with a sedentary lifestyle.

Aim: We hypothesized that regular intake of a snack containing high amounts of soluble fibre in parallel to a regular diet may rapidly improve bowel habits with simultaneous elevation of synthesis of short-chain fatty acids (SCFA).

Material and methods: A total of 20 healthy volunteers, with a stool frequency of less than 3 spontaneous bowel movements per week, completed a 14-day double-blind, parallel-arm, randomized clinical trial with the intervention comprising daily intake of 2 doses $(9.99 \%$ per $100 \mathrm{~g}$ or $13.91 \%$ per $100 \mathrm{~g}$ ) of fibre. Food consumption was evaluated via 72 -hour recall diaries at baseline and after 14 days of intervention. Gastrointestinal symptoms (abdominal comfort, distension, bloating, flatulence, stomach rumbling, number of bowel movements) were monitored via the IBS/VAS scale every day.

Results: We found that the elevated fibre intake improved bowel habits significantly (defecation frequency increased from 0.28 to 0.87 times/day; $p=0.0002$ ) in both study arms. After 14 days of the trial, an increase in SCFA concentration (for whole study group: acetic $p<0.036$, propionic $p<0.019$, and butyric acid $p<0.0012$ compared to baseline parameters) with no adverse gastrointestinal symptoms was reported.

Conclusions: Short-term, regular intake of a snack containing soluble fibre improves the SCFA synthesis and bowel habits in healthy people living a sedentary lifestyle. The exact mechanism behind this observation requires further investigation.
\end{abstract}

\section{Introduction}

Digestive health plays an important role in daily activity of a person throughout their life. Meanwhile, many people experience a variety of gastrointestinal complaints, which in consequence affect their quality of life [1]. Constipation is one of the most common complaints in the general population [2], a multifactorial disorder comprising infrequent bowel movements (usually less than 3 per week) as well as straining, hard stools, and a sense of incomplete defecation [3]. An increased risk of chronic constipation (CC) is seen among individuals with a sedentary lifestyle and persons consuming a low-fibre diet [4]. Epidemiologically, women are affect- ed 5 to 10 times more often than men (female-to-male ratio of $2.2: 1$ ) $[4,5]$.

Dietary fibre is often suggested to maintain proper digestive health [6]. Increasing the intake of dietary fiber is a simple way to regulate the defecation process. Increased fibre intake results in changing the consistency of faeces and increasing the intestinal peristalsis and motility [7]. Dietary fibre is defined as complex carbohydrates undigested with human digestive enzymes [8]. On the other hand, fibre is an energy source for microorganisms living in the intestinal niche. During the microbial fermentation processes, synthesis of several byproducts (e.g., short-chain fatty acids 
(SCFAs) - acetate, propionate, and butyrate predominantly) takes place. Some of them support the health of colonic epithelial cells while the others play other physiological roles, not only in situ [9, 10]. In 2015 Feng et al. [11] showed that the numbers of Bfidobacteria, Lactobacilli, and Methanogens were significantly decreased in the faeces and colonic mucosa of patients after subtotal colectomy due to refractory constipation. The authors depicted substantial differences in the microbiota metabolite pattern, especially the content of SCFA in relation to colonic motility. The following year, the same researchers documented a clear link between the phenotype of constipation, levels of stool SCFAs, and the expression of SCFA receptors (G protein-coupled receptor 43 and choline acetyltransferase) in the colon [12]. Along with these, a recent meta-analysis demonstrated that skewed SCFA content, especially of propionate and butyrate nature, may be the cause of constipation in irritable bowel syndrome (IBS) patients in comparison to healthy persons [9].

\section{Aim}

The aim of our study was to assess whether an increased fibre intake mitigates symptoms of constipation in persons with a sedentary lifestyle who are otherwise healthy. For the purposes of the study we enrolled a group of office workers with average bowel movements less than once every 3 days. The intervention comprised the increase of fibre intake (snacks bars by $9.99 \%$ per $100 \mathrm{~g}$ or $13.91 \%$ per $100 \mathrm{~g}$ of added fibre) in a short (14 days) period. We hypothesized that such a dietary approach would lead to elevation of SCFA synthesis without undesirable side effects, such as gas

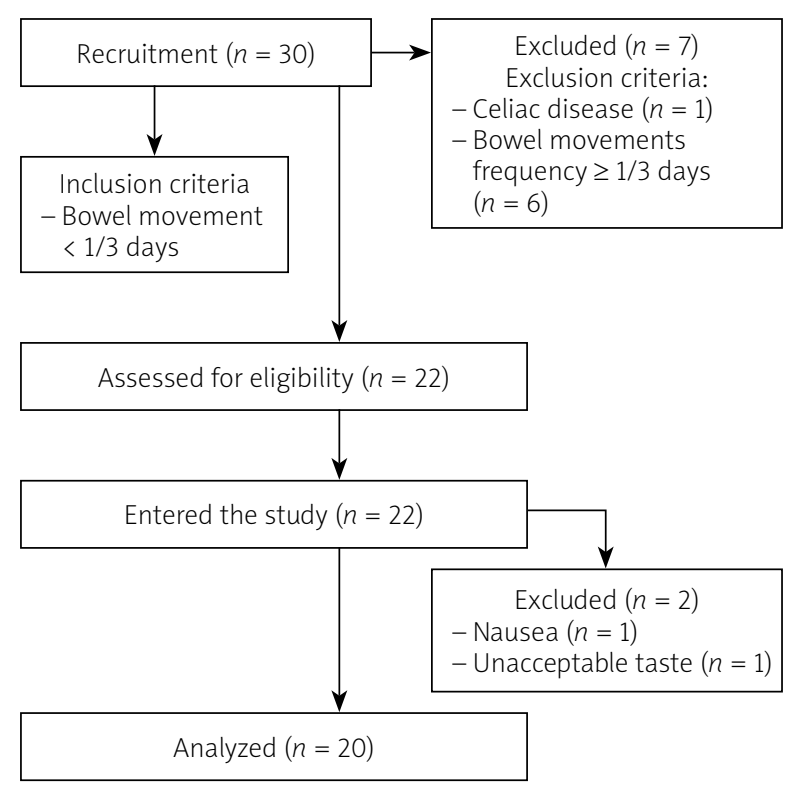

Figure 1. The study flow chart production or abdominal pain, resulting in increased frequency of bowel movements.

\section{Material and methods Participants}

Inclusion criteria were as follows: healthy adults with a stool frequency of less than 3 spontaneous bowel movements per week. The persons were otherwise healthy, without other complaints or with symptoms persisting for the last 3 months. Because strict Rome IV questionnaires were not used, we defined enrolled individuals as those with (CC). Exclusion criteria were as follows: i) change in bodyweight in the 3 months prior to the study; ii) chronic use of antacids, proton pump inhibitors or bulk laxatives; iii) history of major gastrointestinal surgeries; iv) antibiotics or probiotic supplementation in the 3 months preceding the study; v) lack of consent; and vi) a diagnosed psychiatric disorder.

We initially recruited 30 healthy adults for the study, who met the eligibility criteria. Eight individuals were excluded from the study due to normal defecation frequency (more than once every 3 days, $n=6$ ) or a diagnosis of celiac disease $(n=1)$. Finally, 22 people were included in the study. After the intervention, 2 more subjects dropped out of the study (one because of taste unacceptance, the other because of nausea). Finally, there were 20 persons who successfully finished the intervention. The study flow chart is presented in Figure 1.

This project received ethical approval from the Ethics Board of the Pomeranian Medical University (Resolution no. KB-0012/139/18).

\section{Study design}

The study was conducted at the Department of Human Nutrition and Metabolomics of the Pomeranian Medical University in Szczecin between October and November 2019. This was a 14-day, single-centre, placebo-controlled, double-blind study. Participants were randomly assigned to 1 of 2 groups, receiving 1 snack (100 g) per day, comprising 1 of the following isocaloric (257 kcal/100g) snack bars containing a higher or lower amount of fibre: Variant 1 - high-fibre bar contained $13.91 \%$ fibreper $100 \mathrm{~g}$, whilst the content of low-fibre Variant 2 (Control bar) was $9.99 \%$ per $100 \mathrm{~g}$. The composition of the bar was tested in samples of different batches of bars in an accredited external laboratory with PCA (1095) and ILAC MRA certification (Jars S.A, Poland)

Randomization was carried out using researcher randomizer computer software (https://www.randomizer.org/). An investigator who was not involved with the study generated the randomization sequence. Subjects and study staff were blinded to the treatments, and the 
bars were supplied in identical white paper packets. Subjects consumed the bars as a replacement for their current snacks. One bar was consumed per day in all groups to avoid gastrointestinal discomfort, as a meal replacement with a glass $(250 \mathrm{ml})$ of water or tea. All the patients returned all empty packages to assess compliance and actual intake after 7 and 14 days of observation. The nutritional values of bars used within our study are presented in Table I. Clinical examinations, anthropometric body composition measurements, and stool sampling were performed at the start of the study and at the end-point. Body composition measurements were performed using a Tanita scan (Hologic QDR 4500, Hologic, Inc., Bedford, MA, USA).

\section{Nutritional self-reported food intake and gastrointestinal symptoms}

Aside from the study bars, participants purchased all their own food and were instructed to maintain their current level of physical activity. Food pattern was controlled by 72 -h diet recall forms, which were collected after 7 and 14 days of intervention.

Gastrointestinal (GI) symptoms (abdominal comfort, distension, bloating, flatulence, stomach rumbling, number of bowel movements) were monitored via the IBS VAS scale every day [13], following the instruction of the authors' questionnaire, regarding the data collection as well as calculating the results.

\section{Collection of faeces}

Freshly evacuated faeces were collected using a stool kit provided by the investigators (Kalszyk, Poland). Subjects placed their stool sample (at the beginning of the study and at the end of the study (1-2 days after the finish of consumption of the bars) and delivering it to the laboratory within $24 \mathrm{~h}$. Samples were stored at $-80^{\circ} \mathrm{C}$ until analysis.

\section{Isolation and measurement of SCFAs by gas chromatography}

First a $0.5 \mathrm{~g}$ faecal sample was suspended in a tube containing $5 \mathrm{ml}$ of water and mixed intensively for $5 \mathrm{~min}$. Then the $\mathrm{pH}$ was adjusted to $2-3$ with a $5 \mathrm{M} \mathrm{HCl}$ solution. Then samples were shaken for $10 \mathrm{~min}$ and centrifuged for $20 \mathrm{~min}$ at $5000 \mathrm{rpm}$. After that, the supernatant was filtered ( $\varnothing 400 \mu \mathrm{m})$. Chromatographic analyses with fused-silica capillary column (DB-FFAP, $30 \mathrm{~m} \times 0.53 \mathrm{~mm} \times 0.5 \mu \mathrm{m}$ ) were carried out using the Agilent Technologies 1260 A GC system (with a flame ionization detector (FID). The carrier gas was hydrogen at a flow rate of $14.4 \mathrm{ml} / \mathrm{min}$. The initial temperature was $100^{\circ} \mathrm{C}$ (maintained for $0.5 \mathrm{~min}$ ), then raised to
Table I. Nutritional values of bars used in the study

\begin{tabular}{lcc} 
Bar ingredients & Variant 1 & Variant 2 \\
\hline Fibre (\%) & 13.91 & 9.99 \\
\hline Protein (\%) & 6.63 & 7.79 \\
\hline Total carbohydrates (\%) & 15.99 & 13.81 \\
\hline Digestible carbohydrates (\%) & 39.56 & 44.33 \\
\hline Total fats (\%) & 4.95 & 5.45 \\
\hline Saturated fatty acids (g/100 g) & 0.82 & 1.05 \\
\hline Sodium [mg/kg] & 72.2 & 75.5 \\
\hline NaCl (\%) & 0.02 & 0.02 \\
\hline Ash (\%) & 1.70 & 1.60 \\
\hline Dry weight (\%) & 66.95 & 69.16
\end{tabular}

Energy value (KJ/kcal/100g) - variant 1 bar (1080/257); variant 2 bar (1168/278). The sources of fibre in the snack bars were nuts, linseed, oatmeal, and fruit pectin.

$180^{\circ} \mathrm{C}$ (ramping of $8^{\circ} \mathrm{C} / \mathrm{min}$, maintained for $1 \mathrm{~min}$, then to $200^{\circ} \mathrm{C}$ (ramping $20^{\circ} \mathrm{C} / \mathrm{min}$ ), to finally reach $200^{\circ} \mathrm{C}$ and be sustained for $5 \mathrm{~min}$.

\section{Statistical analysis}

Due to the abnormal distribution of study variables, we applied nonparametric tests, namely the Wilcoxon-signed rank and U Mann-Whitney tests. The median and IQR were presented as descriptive statistics. MedCalc ver.19.1.5 (Ostend, Belgia) was used for all statistical analyses. We adopted $p$-value $<0.05$ as statistically significant.

\section{Results}

\section{Subjects' characteristics}

The study comprised a group of 20 healthy individuals with a stool frequency of less than 3 spontaneous bowel movements per week, with female predominance $(n=19)$, aged $40.6 \pm 5.4$ years. At baseline and after 4 weeks the subjects underwent bioimpedance analysis to measure fat mass, lean mass, and percentage body fat. Data regarding basic anthropometric indices are presented in Table II. There were no significant differences regarding these variables throughout the study period.

\section{SCFAs synthesis}

Elevated fibre intake caused significant changes in the content of SCFAs. The overall SCFA pool increased significantly, in particular acetic (C:2), propionic (C:3), butyric (C:4), C5:0, and C6:0, as shown in Table III. 
Table II. Baseline and endpoint characteristics of enrolled patients with a stool frequency of less than 3 spontaneous bowel movements per week

\begin{tabular}{lcccccc} 
Variable & \multicolumn{2}{c}{ Pre intervention } & \multicolumn{2}{c}{ Post intervention } & \multicolumn{2}{c}{ Paired differences } \\
\cline { 2 - 7 } & Median & IQR & Median & IQR & Median & $\boldsymbol{P}_{\text {-value* }}$ \\
\hline BMI $\left[\mathrm{kg} / \mathrm{m}^{2}\right]$ & 27.6 & 7.4 & 27.1 & 8.0 & 0.0 & 0.5 \\
\hline Fat mass (\%) & 32.6 & 18.1 & 3.3 & 10.15 & 0.0 & 0.9 \\
\hline Lean mass (\%) & 47.3 & 7.65 & 46.2 & 8.1 & 0.0 & 0.1 \\
\hline Total body water (\%) & 46.8 & 11.05 & 48.1 & 8.98 & 0.2
\end{tabular}

*Wilcoxon signed rank test, IQR - interquartile range.

Next, we studied whether the content of fibre in the bars might have affected the synthesis of SCFAs. We found that the more fibre was placed in a bar, the more pronounced the fatty acid change was, but these differences were not statistically significant. Due to the low statistical power of the study, i.e., below the recommended value of 0.8 , we can assume that the increased number of participants would justify this issue (Table IV).

\section{Gastrointestinal symptoms}

When analysing a 10-degree VAS-IBS scale we found no statistical differences in the intensity of abdominal pain (before: 0.5 , after: $0.583 ; p=0.75$ ), flatulence (before: 1.556 , after: $0.833 ; p=0.44$ ), gases (before: 1.778, after: $1.083 ; p=0.41$ ), nausea (before: 0.0 , after: $0.556 ; p=0.11$ ), belching (before: 0.5 , after: $0.056 ; p=$ 0.01 ), and the feeling of incomplete bowel movement

Table III. Concentration of SCFAs measured before and after intervention

\begin{tabular}{|c|c|c|c|c|c|}
\hline \multirow[t]{2}{*}{ SCFA $[\mu \mathrm{mol} / \mathrm{g}]$} & \multicolumn{2}{|c|}{ Before the intervention $(n=20)$} & \multicolumn{2}{|c|}{ After the intervention $(n=20)$} & \multirow[t]{2}{*}{$P$-value* } \\
\hline & Median & IQR & Median & IQR & \\
\hline$C 2: 0$ & 99.6 & 63.2 & 137.6 & 109.09 & 0.00 \\
\hline C3:0 & 56.9 & 33.1 & 84.2 & 61.49 & 0.02 \\
\hline$C 4: 0 i$ & 13.5 & 4.5 & 19.3 & 16.36 & 0.05 \\
\hline C4:0 n & 58.4 & 39.9 & 137.9 & 132.46 & 0.00 \\
\hline C5:0 i & 25.5 & 11.5 & 32.10 & 26.04 & 0.12 \\
\hline C5:0 n & 14.2 & 6.80 & 24.4 & 23.83 & 0.01 \\
\hline C6:0 i & 1.40 & 2.00 & 1.90 & 3.37 & 0.43 \\
\hline C6:0 n & 3.50 & 9.10 & 17.3 & 34.26 & 0.01 \\
\hline Total & 297.1 & 96.3 & 500.6 & 395.59 & 0.00 \\
\hline
\end{tabular}

*Wilcoxon signed rank test, IQR - interquartile range.

Table IV. Changes (endpoint vs. baseline) in concentration of SCFAs depending on the type of intervention

\begin{tabular}{|c|c|c|c|c|c|}
\hline \multirow[t]{2}{*}{ SCFA $[\mu \mathrm{mol} / \mathrm{g}]$} & \multicolumn{2}{|c|}{ BAR $13.91 \mathrm{~g}$ fibre $(n=11)$} & \multicolumn{2}{|c|}{ BAR $9.99 \mathrm{~g}$ fibre $(n=9)$} & \multirow[t]{2}{*}{$P$-value* } \\
\hline & Median & IQR & Median & IQR & \\
\hline$C 2: 0$ & 44.08 & 96.86 & 20.42 & 82.57 & 0.47 \\
\hline C3:0 & 21.99 & 52.19 & 15.68 & 85.22 & 0.91 \\
\hline$C 4: 0 i$ & 6.57 & 17.63 & -0.399 & 14.35 & 0.52 \\
\hline C4:0 n & 89.93 & 112.48 & 31.42 & 110.03 & 0.21 \\
\hline C5:0 i & 9.99 & 32.08 & -4.90 & 21.22 & 0.47 \\
\hline C5:0 n & 16.84 & 35.63 & 1.07 & 23.54 & 0.27 \\
\hline C6:0 i & -0.048 & 2.65 & -0.03 & 5.11 & 0.85 \\
\hline C6:0 n & 16.05 & 44.01 & 3.16 & 12.43 & 0.14 \\
\hline Total & 234.94 & 281.06 & 125.91 & 343.82 & 0.47 \\
\hline
\end{tabular}


(before: 0.33, after: 0.33; $p>0.99$ ). Vomiting was not observed in any of the groups at baseline or at the endpoint. These parameters did not change regardless the amount of fibre intake $(p=0.96 ; p=0.96 ; p=0.19$; $p=0.83 ; p=0.8 ; p=0.44$, respectively).

The average number of bowel movements before the intervention was 0.28 (2 per week) and after 14 days of the study it was 0.872 ( $>2$ per week). The difference was statistically significant $(p=0.0002)$. However, there were no statistically significant differences in the bowel movements between the groups consuming bars with $13.91 \%$ of fibre per $100 \mathrm{~g}$ (average 0.603 ) and $9.99 \%$ of fibre per $100 \mathrm{~g}$ (average 0.574 ).

\section{Diet content}

In both groups macronutrient intake was strictly controlled and was not significantly different compared to baseline variables, except protein and fibre intake, as shown in Table V. As expected, we observed a lower intake of protein and higher intake of fibre after 14 days of the study.

\section{Discussion}

In our study we indirectly searched for a link between microbiota byproducts - SCFAs - and constipation. We observed an increase in the average number of bowel movements after 14 days of consumption of a snack containing soluble fibre. The difference was statistically significant. CC is a disorder with multifactorial and poorly understood aetiology. Several studies have proven that the microbiota composition in patients with CC was altered in comparison to heathy controls $[14,15]$. Therefore, constipation should be vulnerable to a dietary approach because the diet is the strongest factor contributing to the gut ecosystem composition [16]. For instance, in a study by Zhu et al. stool samples collected from young patients (aged 35 years on average) with CC contained significantly lower counts of Bifidobacterium and Bacteroides [14]. A short-term (2 weeks) implemen- tation of a probiotic VSL\#3 improved clinical symptoms of patients diagnosed with functional constipation (FC). In contrast, Parthasarathy et al. [17] demonstrated no difference in the abundance of particular bacteria at the genus level in stools from patients with less than 3 bowel movements per week (aged 48 years on average) and healthy control groups. However, it has to be underlined that the constipated group in this study included individuals diagnosed with constipation predominant or mixed type - IBS; thus, the results could were biased by disease $p$ phenotype [17]. Last year, Okhusa et al. [18] elegantly concluded that the constipation-type microbiota may be modulated with prebiotics and probiotics or other microbiota-based interventions.

One of the most tested prebiotics, inulin, has its own "activity" on bowel motility. Also, it was shown that such a prebiotic supply had a strong impact on human gut microbiota. A recent systematic review demonstrated that inulin was able to increase the abundance of Bifidobacterium, Anaerostipes, Faecalibacterium, and Lactobacillus parallelly with the decrease of Bacteroides [19]. Therefore, the hypothesis adopted by our team in the present study was that via modulation of gut microbiota composition along with its fermentation activity (SCFAs), due to increased fibre intake may improve the frequency of bowel movements. Such a strategy is a relatively simple and novel approach; to the best of our knowledge, no other intervention comprising prebiotic supply in patients reporting less than 3 bowel movements per week with no other symptoms exists in Poland.

We were able to demonstrate that a prebiotic supply elevated the content of SCFA in the stools of people with chronic constipation in comparison to a healthy group of Polish women without any defecation problems. As presented in a previous study by Skonieczna-Zydecka et al. [20], the median concentration of acetic acid (C:2) in stool reached $28.49 \mu \mathrm{mol} / \mathrm{g}$ whereas in our group (at the study beginning) it was equal to

Table V. The intake of nutrients before and after the prebiotic intervention

\begin{tabular}{|c|c|c|c|c|c|}
\hline \multirow[t]{2}{*}{ Variable } & \multicolumn{2}{|c|}{ Before $(n=16)$} & \multicolumn{2}{|c|}{ After $(n=9)$} & \multirow[t]{2}{*}{$P$-value* } \\
\hline & Median & IQR & Median & IQR & \\
\hline Energy [kcal] & 1758.16 & 534.94 & 1646.82 & 554.39 & 0.11 \\
\hline Water [g] & 1943.31 & 951.86 & 1829.03 & 1482.53 & 0.25 \\
\hline Protein [g] & 94.22 & 20.24 & 71.15 & 18.36 & 0.00 \\
\hline Fat [g] & 55.15 & 25.08 & 55.97 & 36.00 & 0.31 \\
\hline Carbohydrates [g] & 225.84 & 98.21 & 214.40 & 76.07 & 0.86 \\
\hline Fibre $[\mathrm{g}]$ & 15.27 & 4.87 & 25.23 & 11.08 & 0.02 \\
\hline
\end{tabular}


$99.6 \mu \mathrm{mol} / \mathrm{g}$. Similarly, other SCFA concentrations in stools of our participants were higher (compared to the study by Skonieczna-Zydecka et al. [20]). Consequently, we observed higher concentration of $\mathrm{C}: 3$ (propionate: $16.9 \mu \mathrm{mol} / \mathrm{g}$ vs. $56.9 \mu \mathrm{mol} / \mathrm{g}$ in our study), C 4:0 I (isobutryrate: $4.7 \mu \mathrm{mol} / \mathrm{g}$ vs. $13.5 \mu \mathrm{mol} / \mathrm{g}$ in our study), and C 4:0 n (butrytate: $20.8 \mu \mathrm{mol} / \mathrm{g}$ vs. $58.4 \mu \mathrm{mol} / \mathrm{g}$ in our study), as well C 5:0 i (isovaleriate $0.9 \mu \mathrm{mol} / \mathrm{g}$ vs. $25.5 \mu \mathrm{mol} / \mathrm{g}$ in our study); C:5 n (valeriate $5.7 \mu \mathrm{mol} / \mathrm{g}$ vs. $14.2 \mu \mathrm{mol} / \mathrm{g}$ in our study), and C 6:0 (3.2 $\mu \mathrm{mol} / \mathrm{g}$ vs. $3.5 \mathrm{~mol} / \mathrm{g}$ in our study). It seems that the higher SCFA content observed among constipated patients may result from SCFA accumulation. This phenomenon may be caused by limited transit time of the stool, and thus elevated bloom of gut microbes, but this result requires further research including gut microbiome analysis.

Different results were obtained by Kang et al. [21], who studied healthy subjects (with normal bowel habits) and subjects with FC. The total SCFA content did not differ between groups, but they found that iso-butyrate $(C: 4 i)$ was significantly higher in stool samples of constipated subjects compared with healthy subjects. However, it is difficult to compare our results to those of Kang et al. [21] because a different method for SCFA analysis - HPLC - was used in their work, while gas chromatography was used in the present paper.

Our results are also partly different to the results published recently by Chu et al. [22]. This Korean research team supplemented chronically constipated adults with prebiotics containing non-digestible carbohydrates: inulin, lactitol, and aloe vera gel for 4 weeks. Next, the authors analysed the gut microbiota composition, SCFA production, and serum endotoxemia markers. After the intervention with prebiotics, in contrast to our current study, Chu et al. did not observe any significant differences in SCFA concentration in stool. It is worth emphasizing that in parallel the study demonstrated significantly increased abundance of the faecal butyrate producing bacterium Roseburia hominis and reported improved stool frequency. The authors also noticed a positive association between the dietary intervention and bacterial lipopolysaccharide content and its receptor CD14 (in serum). Chu et al. [22] used prebiotic UG1601 (soluble fibre in gel form) whereas we used mixed fibre with soluble and non-soluble components; these may explain the differences observed in our trial.

In our study an increased intake of fibre led to elevation of the synthesis of almost all SCFAs and accelerated the intestinal peristalsis without unpleasant gastric symptoms (bloating, pain, gas, belching). In the American study a group of 49 healthy men and 32 postmenopausal women provided with a short-term consumption of fibres from whole grain achieved pos- itive effects regarding SCFA concentration and stool frequency [23].

One could ask how SCFAs the improvement of bowel movement frequency among our patients - the data in the literature states that SCFAs could play a positive role in the regulation of frequency of bowel movements by direct effect on stool bulkiness, $\mathrm{pH}$ of the stool, and intestinal transit time [24]. The essential SCFAs released through fermentation of resistant starches and fibre are C:2 (acetate), C:3 (propionate), and C:4 (butyrate), and their molar ratio of production in the colon is $60: 25: 15$ [9]. SCFA metabolic functions include the following: a) they act as factors reducing the $\mathrm{pH}$ in the colon and in this way cause removal of bacterial pathogens, b) they regulate the metabolism of colonocytes and the immune system, c) they increase the tightness of the intestinal barrier through specific receptors for SCFA (GPR41 (FFAR3), GPR43 (FFAR2), GPR109a (PUMA-G) [25]), d) they reduce endotoxaemia (associated with dysbiosis), and e) they alter chemotaxis and phagocytosis [9], all of which may lead to relief of constipation symptoms [22].

Changes in the concentrations of colonic SCFAs is a process that is difficult to understand because of its high complexity and dynamics. The sensitive interaction between the colon and microbiota may also control the proportion and levels of SCFAs in the gut lumen [9].

Acetic acid (C 2:0) is a source of energy. In the liver, acetate and butyrate are lipogenic [26]. Acetate (as well as propionate and butyrate) appears to regulate hepatic lipid and glucose homeostasis [26]. C:3 (propionate) has been shown as a factor that mitigates the response of innate immune cells toward bacterial stimulation; therefore, a higher content of C: 3 in the gut may be due to its relatively weak anti-inflammatory activity and incorrect permeability of the intestinal barrier [27]. A meta-analysis published recently reported the differences between $\mathrm{C}: 2$ and $\mathrm{C}: 3$ concentrations in patients with constipation type irritable bowel syndrome (IBS-C) and chronic constipation [9]. In IBS-C patients, both propionate and butyrate were reduced in comparison to CC [9].

C:4 (butyrate) is the preferred fuel utilized by coloncytes [26]. The metabolite plays a pivotal role in maintaining the gut-barrier function by enhancing mucin secretion as well as the stabilization of tight junction proteins, reducing the translocation of inflammatory agents (LPS) [28]. Butyrate acts also as a histone deacetylase (HDAC) inhibitor and a ligand for a subset of G protein-coupled receptors [29].

The data on the physiological role of the other two SCFAs: valeric (C5:0) and caproic acid (C6:0) are scarce. C6:0 acid is known as a biomarker of Clostridium difficile presence within the gut microbiota in the infant 
gut. It is worth noting that even a small increase in the daily fibre portion resulted in the regulation of bowel movements. Interestingly, based on 72-h recall diaries, patients ate less protein during the study. Reducing the amount of these macronutrients was reported to reduce the amount of putrefactive microbiota in the intestine, which has a positive effect on metabolism.

Strengths: easy to implement protocol. Limitations: lack of microbiota analyses, lack of assessment of gut barrier markers.

\section{Conclusions}

Mixed fibre added to the regular diet for a minimum of 14 days improved the bowel movements in persons with CC along with modification of stool SCFA content.

\section{Acknowledgments}

"Inkubator Innowacyjności +" project of the Maremed consortium (Pomeranian Medical University Technology Transfer Centre and Innovation Centre of the Maritime University Ltd.) with financial support from the Ministry of Science and Higher Education and EU funds (ERDF).

\section{Conflict of interest}

The authors declare no conflict of interest.

\section{References}

1. Canavan C, West J, Card T. Change in quality of life for patients with irritable bowel syndrome following referral to a gastroenterologist: a cohort study. PLoS One 2015; 10: e0139389.

2. Costilla VC, Foxx-Orenstein AE. Constipation: understanding mechanisms and management. Clin Geriatr Med 2014; 30: 107-15.

3. Rasmussen HE, Hamaker B, Rajan KB, et al. Starch-entrapped microsphere fibers improve bowel habit but do not exhibit prebiotic capacity in those with unsatisfactory bowel habits: a phase I, randomized, double-blind, controlled human trial. Nutr Res 2017; 44: 27-37.

4. Alame AM, Bahna H. Evaluation of constipation. Clin Colon Rectal Surg 2012; 25: 5-11.

5. Higgins PDR, Johanson JF. Epidemiology of constipation in North America: a systematic review. Am J Gastroenterol 2004; 99: 750-9.

6. Dreher ML. Whole fruits and fruit fiber emerging health effects. Nutrients 2018; 10: 1833.

7. Christodoulides S, Dimidi E, Fragkos KC, et al. Systematic review with meta-analysis: effect of fibre supplementation on chronic idiopathic constipation in adults. Aliment Pharmacol Ther 2016; 44: 103-16.

8. Rao TP, Quartarone G. Role of guar fiber in improving digestive health and function. Nutrition 2019; 59: 158-69.

9. Sun Q, Jia Q, Song L, Duan L. Alterations in fecal short-chain fatty acids in patients with irritable bowel syndrome: a sys- tematic review and meta-analysis. Medicine 2019; 98: e14513.

10. Dalile B, Van Oudenhove L, Vervliet B, Verbeke K. The role of short-chain fatty acids in microbiota-gut-brain communication. Nat Rev Gastroenterol Hepatol 2019; 16: 461-78.

11. Feng X, Su Y, Jiang J, et al. Changes in fecal and colonic mucosal microbiota of patients with refractory constipation after a subtotal colectomy. Am Surg 2015; 81: 198-206.

12. Shi Y, Chen Q, Huang Y, et al. Function and clinical implications of short-chain fatty acids in patients with mixed refractory constipation. Colorectal Dis 2016; 18: 803-10.

13. Bengtsson M, Ohlsson B, Ulander K. Development and psychometric testing of the Visual Analogue Scale for Irritable Bowel Syndrome (VAS-IBS). BMC Gastroenterol 2007; 7: 16.

14. Zhu L, Liu W, Alkhouri R, et al. Structural changes in the gut microbiome of constipated patients. Physiol Genomics 2014; 46: 679-86.

15. Zhao Y, Yu YB. Intestinal microbiota and chronic constipation. Springerplus 2016; 5: 1130.

16. Zmora N, Suez J, Elinav E. You are what you eat: diet, health and the gut microbiota. Nat Rev Gastroenterol Hepatol 2019; 16: 35-56.

17. Parthasarathy G, Chen J, Chen X, et al. Relationship between microbiota of the colonic mucosa vs feces and symptoms, colonic transit, and methane production in female patients with chronic constipation. Gastroenterology 2016; 150: 367-79.

18. Ohkusa T, Koido S, Nishikawa Y, Sato N. Gut microbiota and chronic constipation: a review and update. Front Med 2019; 6: 19.

19. Le Bastard Q, Chapelet G, Javaudin F, et al. The effects of inulin on gut microbial composition: a systematic review of evidence from human studies. Eur J Clin Microbiol Infect Dis 2020; 39: 403-13.

20. Skonieczna-Żydecka K, Grochans E, Maciejewska D, et al. Faecal short chain fatty acids profile is changed in Polish depressive women. Nutrients 2018; 10: 1939.

21. Kang DW, DiBaise JK, Ilhan ZE, et al. Gut microbial and shortchain fatty acid profiles in adults with chronic constipation before and after treatment with lubiprostone. Anaerobe 2015; 33: 33-41.

22. Chu JR, Kang SY, Kim SE, et al. Prebiotic UG1601 mitigates constipation-related events in association with gut microbiota: a randomized placebo-controlled intervention study. World J Gastroenterol 2019; 25: 6129-44.

23. Vanegas SM, Meydani M, Barnett JB, et al. Substituting whole grains for refined grains in a 6-wk randomized trial has a modest effect on gut microbiota and immune and inflammatory markers of healthy adults. Am J Clin Nutr 2017; 105: 635-50.

24. Adams S, Che D, Qin G, et al. Interactions of dietary fibre with nutritional components on gut microbial composition, function and health in monogastrics. Curr Protein Pept Sci 2018; 19: 1011-23.

25. Marangoni F, Martini D, Scaglioni S, et al. Snacking in nutrition and health. Int J Food Sci Nutr 2019; 70: 909-23.

26. Morrison DJ, Preston T. Formation of short chain fatty acids by the gut microbiota and their impact on human metabolism. Gut Microbes 2016; 7: 189-200. 
27. Ciarlo E, Heinonen T, Herderschee J, et al. Impact of the microbial derived short chain fatty acid propionate on host susceptibility to bacterial and fungal infections in vivo. Sci Rep 2016; 6: 37944

28. Yamawaki Y, Yoshioka N, Nozaki K, et al. Sodium butyrate abolishes lipopolysaccharide-induced depression-like behaviors and hippocampal microglial activation in mice. Brain Res 2018; 1680: 13-38.

29. Yao CK, Muir JG, Gibson PR. Review article: insights into colonic protein fermentation, its modulation and potential health implications. Aliment Pharmacol Ther 2016; 43: 181-96.

Received: 6.01.2021

Accepted: 16.03 .2021 